\title{
La ciudad de las oportunidades. Turistificación y emprendimientos del Centro Histórico de Quito
}

\section{The city of opportunities. Turistification and entrepreneurship of the Historic Center of Quito}

\author{
Víctor Elías Llugsha Guijarro \\ Universidad UTE, Ecuador
}

Autor para correspondencia: vikxox@gmail.com

Fecha de recepción: 12 de julio del 2019 - Fecha de aceptación: 05 de noviembre del 2019

\section{Resumen}

La conservación del patrimonio del Centro Histórico de Quito se encuentra relacionado de forma próxima a las actividades turísticas. La peatonización de varias calles y la creación de terrazas turísticas de este espacio, se justifican en criterios de seguridad, disminución de los niveles de contaminación ambiental, el mejoramiento de la calidad del aire y brindar mejores condiciones para la llegada de turistas. El documento analiza la relación existente entre patrimonialización y turismo, tomando en cuenta las acciones sobre el territorio y los actores que se benefician de las condiciones que el destino les ofrece, remplazando actividades económicas tradicionales, por nuevas que responden a un enfoque de boutiquización (hoteles, restaurantes, tiendas de recuerdos). Se abordan los conflictos que se generan alrededor de la turistificación del Centro Histórico de Quito, donde la construcción de mejores espacios para el desarrollo de actividades turísticas, llevan a la ciudad a procesos de cambios de actividades económicas y aceleración de la pérdida de población local.

Palabras clave: turismo; emprendimiento; turistificación; centralidad; boutiquización; turismo urbano

\begin{abstract}
The conservation of the heritage of the Historic Center of Quito is closely related to tourism activities. The pedestrianization of several streets and the creation of tourist terraces of this space, are justified in safety criteria, decrease of the levels of environmental pollution, the improvement of the air quality and provide better conditions for the arrival of tourists. The document analyzes the relationship between patrimonialization and tourism, taking into account the actions on the territory and the actors that benefit from the conditions that the destination offers them, replacing traditional economic activities, with new ones that respond to a boutiquization approach (hotels, restaurants, souvenir shops). The conflicts that are generated around the tourism of the Historic Center of Quito are addressed, where the construction of better spaces for the development of tourist activities, lead the city to processes of changes in economic activities and acceleration of the loss of local population.
\end{abstract}

Key words: tourism; entrepreneurship; turistification; centrality; boutiquization; urban tourism 


\section{Introducción}

Los modelos turísticos (comportamiento y oferta turística similar) pueden ser de dos tipos: un modelo segregado o de enclaves, donde el destino es de bajo costo, la población local es parte de puestos operativos y la inversión de las empresas es externa a la comunidad. O un modelo integrado, cuando la experiencia turística está vinculada al ambiente y la localidad, la comunidad es parte de los procesos de planificación mediante la asociación de pequeñas y medianas empresas con un sólido capital social (Inostroza, 2008).

En ambos casos las actividades turísticas abarcan ámbitos sociales, culturales y, ambientales, sin embargo su principal foco está centrado en el desarrollo económico, donde los agentes que tienen más poder de acción definen la dirección del desarrollo de las actividades turísticas, siendo los colectivos más frágiles los que terminan siendo suprimidos (Oliveira \& Pimentel, 2016).

Las investigaciones sobre turismo han abordado este tipo de fenómenos, considerando desde distintas entradas las transformaciones y consecuencias que las actividades turísticas generan en los territorios (Llugsha, 2019, p. 14), “ante la necesidad de proteger los recursos territoriales turísticos de situaciones de agotamiento, que aportan inseguridad a las inversiones y al crecimiento de la propia estructura productiva” (Martínez del Vas, 2017, p. 440), buscando evitar un desarrollismo turístico sin desarrollo (Moreno, 2014).

Las ciudades son los principales polos de atracción de los turistas, debido a que albergan gran parte del patrimonio cultural y cuentan con infraestructura de comunicación y movilidad. Alrededor de la carga histórica de sus sitios fundacionales (centros históricos), se realizan actividades económicas, políticas y de habitabilidad, que en mayor o menor medida que se desarrollan en el espacio público permiten a la ciudad ser el espacio de encuentro de los distintos actores que se mantienen en una continua disputa por el patrimonio y la patrimonialización.

La patrimonialización es un proceso en que está fuertemente ligado a la sacralización de la externalidad cultural, donde se define un tipo de ideal cultural global; y la puesta en valor o activación que dependen fundamentalmente de poderes políticos y las negociaciones que mantienen con la sociedad y los poderes fácticos (Prats, 2005). Se legitima la noción clásica de patrimonio, donde es importante conservar la ciudad histórica por su carga de tiempo (historia) y su importancia universal (Durán, 2014). Este es un proceso que entre las décadas de 1970 a 1990 plantea la necesidad de patrimonializar los centros históricos, a través de una serie de iniciativas que sumado a la participación de la UNESCO, dará como resultado las declaratorias de Patrimonio Cultural de la Humanidad (Verena, 2017).

Este patrimonio nace y se desarrolla en medio de una relación social específica, donde los sujetos que reconoce, se apropian o protegen el patrimonio toman la categoría se sujetos patrimoniales, existen varios criterios que permiten la identificarlos: por el ámbito (local, nacional, provincial o internacional), por su origen (público, privado o comunitario), por su función (comercial, administrativa o de servicios) (Carrión, 2003, p. 141) . 
El documento toma como premisa el hecho de que el modelo de ciudad turística prioriza la utilización del patrimonio como un elemento de contemplación (no se permite que los actuales actores puedan hacer uso o modificación del patrimonio) y mercantilización donde el patrimonio es valorado en tanto genere algún tipo de retorno económico, el turismo propicia activaciones patrimoniales que responden abiertamente a evaluaciones de "consumo como medidor tanto de la eficiencia política como la contribución al desarrollo o consolidación del mercado lúdicoturístico-cultural" (Prats, 2005), donde los servicios y productos para turistas llegan a utilizar los espacios de la ciudad que han sido intervenidos por el sector público, construyendo una suerte de escaparate en la que se muestra el patrimonio a manera de una boutique en la que las personas pueden en sus vitrinas ser espectadores de lo que se legitima como adecuado de ser mostrado a los visitantes que recorren la ciudad.

En tal sentido el documento tiene como objetivo el análisis de la relación existente entre patrimonialización y turismo, tomando como territorio al Centro Histórico de Quito y los actores que se benefician de la patrimonialización del espacio, considerando que en la ciudad se establecen hoteles boutique, cafeterías boutique, artesanías boutique, bajo la lógica de atender las necesidades de la visita de posibles turistas (Carrión, 2009); es decir, el criterio de habitabilidad (en especial para personas de bajos ingresos) es remplazado por un enfoque comercial de lujo (Delgadillo-Polanco, 2010), dando paso a una ciudad en camino a turistificarse.

La turistificación es un término relativamente nuevo, que busca conceptualizar el fenómeno de la masificación del turismo. Este puede ser visto como un proceso mediante el cual los bienes culturales que han sido patrimonializados se convierten en atractivos turísticos (Menchero, 2017). Esta lógica se enmarca en el hecho de que "la gestión y preservación turístico-patrimonial es una intervención técnica, ideológica y políticamente orientada, en la que se decide qué elementos y tipo de estética mantener” (Verena, 2017).

\section{El Centro Histórico de Quito y sus particularidades}

La ciudad de Quito cuenta con una centralidad histórica que se considera viva, debido a las actividades comerciales y políticas que en el espacio se desarrollan, pero sobretodo porque es un lugar donde todavía existe actividad residencial. Ha conservado bienes muebles e inmuebles que son reconocidos como bienes patrimoniales, que mantienen una relación de uso e interés con la población y el territorio.

Factores como la cercanía a lugares de interés en el Centro Histórico o vivir la experiencia de pernoctar en un sitio Patrimonio Cultural de la Humanidad han concitado el interés de los turistas por vivir la experiencia de la ciudad antigua; con el propósito de incentivar las actividades turísticas en el territorio, el Municipio de Quito ha intervenido el Centro Histórico a distintos niveles y en distintos ámbitos, siendo los procesos de peatonización de varias calles y la implementación de las denominadas terrazas turísticas las acciones más recientes y de mayor visibilización en el espacio.

La peatonización es presentada como la posibilidad de contar con sitios amigables donde los propietarios de establecimientos comerciales pueden realizar inversiones; como parte de este proceso, el Instituto Metropolitano de Patrimonio colocó bancas y maceteros en una sección de 
la calle García Moreno (107.120,00 dólares), buscando “que el paseo peatonal luzca atractivo para los visitantes del casco colonial”, materializando la ideología, estética y actores patrimoniales que pueden hacer uso del espacio.

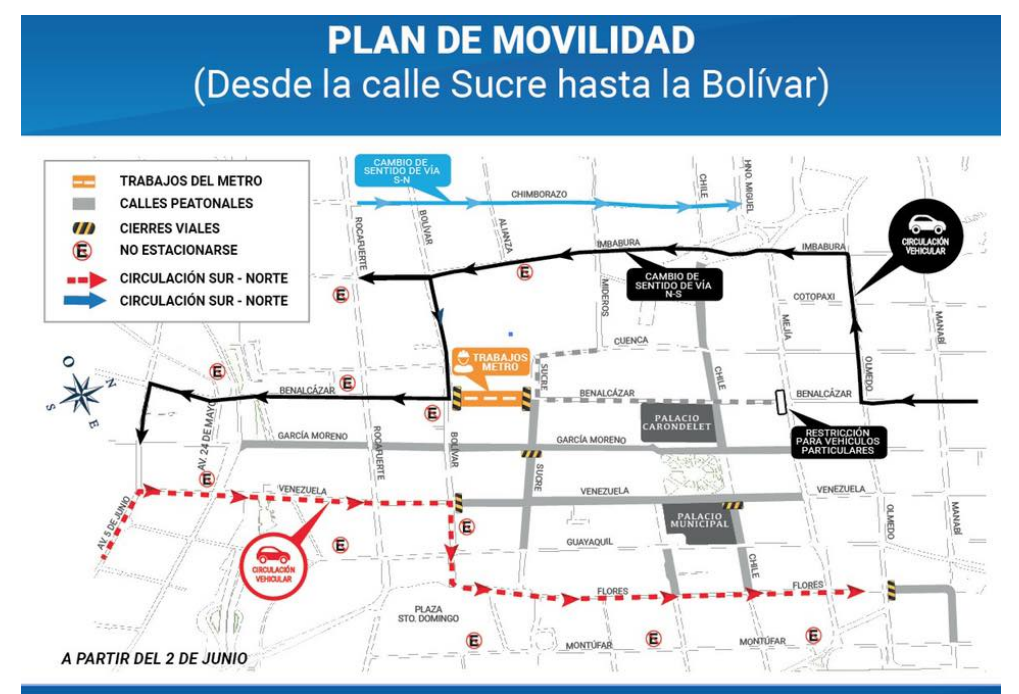

Ilustración \#1 Se describe el plan de movilidad para el Centro Histórico de Quito, a propósito de un cierre vial por la construcción del Metro de Quito. En la imagen se muestran con un color gris las calles peatonizadas del sector.

Fuente: Agencia Metropolitana de Control de Transporte Terrestre, Tránsito y Seguridad Vial, 2019.

En los artículos 1 y 3 de la resolución No. STHV-08-2018, se establece que las terrazas turísticas son espacios complementarios a la actividad comercial de establecimientos cuyo giro de negocios es la venta de alimentos y bebidas, también establecimientos de alojamiento que brinden el servicio de alimentos y bebidas; ambos casos corresponden de forma exclusiva al Centro Histórico, sin embargo no se autoriza a establecimientos que se encuentren al interior de las edificaciones, es decir que para ingresar a estos se debe atravesar patios, galerías o zaguanes.

El Municipio de Quito realizó un proceso de socialización con la comunidad que se desarrolló en tres fases: introductoria, informativas y de asambleas; las tres fases se desarrollaron con el objetivo de informar a residentes y comerciantes para que tomen precauciones y se beneficien posteriormente del proyecto. (Instituto Metropolitano de Patrimonio, 2018). Por otra parte, colectivos como “Defensa del Centro Histórico de Quito”, conformado por moradores del sector cuestionan y rechazan este tipo de intervenciones, más allá de lo estético en beneficio del turismo, mencionan que la peatonización no fue planificada ni organizada.

El principal problema identificado es la disminución de las ventas de sus locales comerciales, problemas de amovilidad y accesibilidad para los residentes, limitación de horarios de servicios; aumento de problemas que en las últimas décadas se han convertido en inherentes al Centro Histórico: venta de drogas, inseguridad, prostitución, ventas ambulantes.

\section{Materiales y métodos}

El documento realiza una exploración de tipo cualitativa, tomando como base fuentes secundarias para abordar los aspectos conceptuales que sustentan la argumentación de los procesos de turistificación y boutiquización de la centralidad histórica. Adicionalmente se 
desarrolló observación participante mediante recorridos por las calles García Moreno, Venezuela, Espejo, Benalcázar, Mejía, Chile y, Junín con el propósito de evidenciar en el territorio el tipo de oferta turística.

Respecto al comportamiento turístico en el territorio, se tomaron en cuenta datos estadísticos de Quito Turismo respecto al número de turistas que llegan a la ciudad y los principales sitios que atraen su visita. Por otra parte, en relación a la planta turística, se expone principalmente al sector de alojamiento, debido a que es una de las actividades económicas ligadas al turismo que mayores impactos genera en los barrios a propósito de la densificación de oferta de hoteles de cinco y cuatro estrellas.

Entre las limitaciones que se presentan en la investigación se encuentra la falta de información estadística actualizada.

\section{Resultados}

Durante el 2018 llegaron a la ciudad 692.492 turistas, lo que representó un crecimiento del 6\% en comparación del año anterior, los sitios más visitados según cifras de Quito Turismo son: Mitad del Mundo, (86\%) Centro Histórico (67\%), Teleférico (12\%), La Mariscal (12\%), El Panecillo (11\%), La Ronda (9\%), Iglesias (11\%); hasta el segundo semestre de ese año se registraron un total de 4381 establecimientos turísticos, de los cuales en su mayoría (64\%) realizan actividades de alimentos y bebidas. La mayor cantidad de establecimientos turísticos se encuentran densificados en dos sectores de la ciudad: el barrio Mariscal Sucre (La Mariscal) y el Centro Histórico, razón por la cual a partir del año 2012 se establecieron las denominadas Zonas Especiales Turísticas (ZET), con el propósito de contar con una normativa especial para estos dos sectores de la ciudad y así poder: regular, controlar y promover el desarrollo de actividades turísticas (Quito Turismo, 2017).

En el proceso de socialización del proceso de peatonización e implementación de terrazas turísticas, el Municipio de Quito realizó 132 encuestas a establecimientos, residencias, locales comerciales de las edificaciones que se encuentran en los tramos que fueron intervenidos. Entre los datos obtenidos se destaca que el 70,5\% de las edificaciones son de uso comercial, el 23,5\% son de uso mixto y, solamente el 1,5\% de las edificaciones son de uso residencial, de las cuales el 71,\% son arrendadas (Instituto Metropolitano de Patrimonio, 2018).

En su planta turística, Quito registra 653 establecimientos de alojamiento, siendo mayoritariamente los establecimientos de dos estrellas (40\%). 


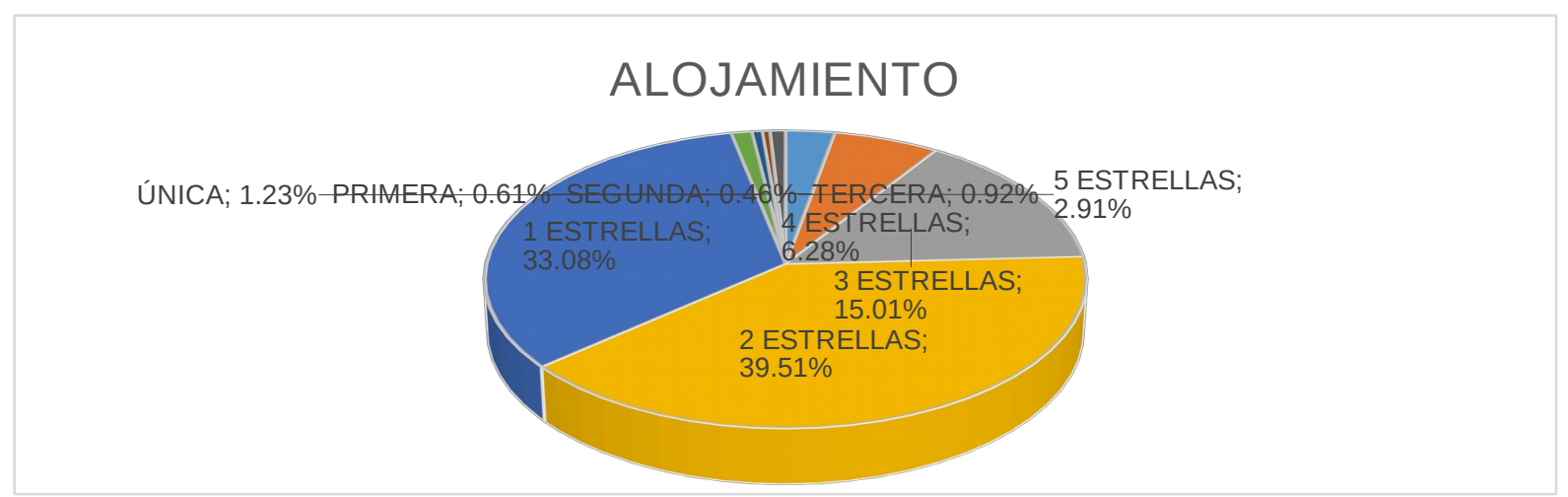

Ilustración \# 2 Se desglosa el sector de alojamiento en Quito, por categoría. Fuente: Quito Turismo, 2017.

Se identificaron distintos tipos de actores patrimoniales: externos a los barrios, grandes inversionistas que buscan atraer un turismo de élite, es así que por ejemplo los establecimientos de hospedaje con cinco y cuatro estrellas que representan el $9 \%$ del total, se concentran en el Centro Histórico de Quito el 27,5\%. Este tipo de oferta ha crecido junto a una oferta de servicios complementarios de tipo lúdico-turístico cultural (tiendas de cacao, café, cerveza artesanal, artesanías, cocina gourmet).

Tabla. 1

Establecimientos de alojamiento de 5 y 4 estrellas en el Centro Histórico de Quito.

\begin{tabular}{ll}
\hline Categoría & \multicolumn{1}{c}{ Establecimientos } \\
\hline \multirow{3}{*}{5 estrellas } & Establecimiento de alojamiento \\
& Plaza Grande \\
& Illa Experience Hotel \\
& Hotel Mama Cuchara \\
\hline \multirow{3}{*}{4 estrellas } & Posada Colonial Colhome \\
& Hotel San Agustín \\
& El Patio Andaluz \\
& El Relicario del Carmen \\
& Boutique Plaza Sucre \\
& Casa Gangotena \\
& Carlota \\
& Hotel Castillo Vista del Ángel \\
\hline
\end{tabular}

Fuente: elaboración propia, (basado en el catastro nacional de la planta turística del Ecuador. Ministerio de Turismo del Ecuador, 2018)

Otro grupo de actores patrimoniales externos identificados son los pequeños inversionistas que rentan locales comerciales, su permanencia en el territorio corresponde a su horario de trabajo y es común que se trasladen de un barrio a otro cuando los propietarios de los locales deciden terminar sus contratos de arrendamiento o no logran conseguir los réditos económicos esperados.

Un tercer grupo de actores patrimoniales identificado, corresponde a los habitantes de los barrios que buscan beneficiarse de las actividades turísticas en su territorio; si bien durante los últimos años han ganado espacio, elementos como su tipo de conformación, número de participantes, experiencia, canales de comercialización, entre otras, han resultado limitantes que no les ha permitido ser protagonistas del turismo en su localidad. 
Tabla.2

Colectivos y asociaciones de turismo comunitario urbano

\begin{tabular}{|c|c|c|c|}
\hline Colectivo/Asociación & Motivación & Particularidades & $\begin{array}{l}\text { Gasto } \\
\text { promedio }\end{array}$ \\
\hline $\begin{array}{l}\text { Caminos de San Roque - } \\
\text { San Roque }\end{array}$ & $\begin{array}{l}\text { Preservación del } \\
\text { patrimonio }\end{array}$ & $\begin{array}{l}\text { Los beneficios económicos de } \\
\text { la asociación son } \\
\text { complementarios a sus } \\
\text { principales actividades } \\
\text { económicas, puesto que las } \\
\text { ganancias generadas por las } \\
\text { actividades turísticas son } \\
\text { mínimas. } \\
\text { Su principal cliente es } \\
\text { Metropolitang Touring, con } \\
\text { quien tienen un contrato de } \\
\text { exclusividad, lo que les impide } \\
\text { poder comercializar con otras } \\
\text { agencias. }\end{array}$ & 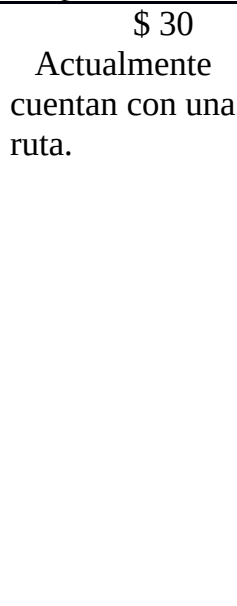 \\
\hline $\begin{array}{l}\text { Colectivo Chakiñán - La } \\
\text { Colmena }\end{array}$ & \begin{tabular}{l}
\multicolumn{1}{c}{ La inseguridad } \\
asociada al barrio los \\
muestra como actores \\
desconectados del \\
patrimonio
\end{tabular} & $\begin{array}{l}\text { Los réditos económicos son } \\
\text { direccionados hacia miembros de } \\
\text { la comunidad que cuentan con } \\
\text { espacios para uso turístico, los } \\
\text { guías turísticos deben pertenecer } \\
\text { al barrio. } \\
\text { No les interesa comercializar } \\
\text { sus productos con agencias de } \\
\text { viajes u operadoras turísticas. }\end{array}$ & $\begin{array}{l}\qquad \$ 20 \\
\text { Las personas } \\
\text { pueden optar } \\
\text { por cinco } \\
\text { distintas rutas }\end{array}$ \\
\hline $\begin{array}{l}\text { Comité de emprendedores } \\
\text { San Marcos }\end{array}$ & $\begin{array}{l}\text { Promover la cultura y } \\
\text { el turismo en el barrio }\end{array}$ & $\begin{array}{l}\text { Las ganancias son compartidas } \\
\text { con los lugares que forman parte } \\
\text { de la ruta. } \\
\text { Ninguna agencia de viajes } \\
\text { comercializa sus productos }\end{array}$ & 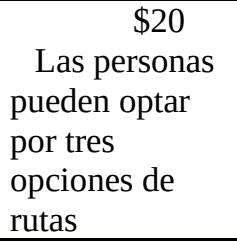 \\
\hline
\end{tabular}

Fuente: elaboración propia (basado en Larrea, 2019)

Una forma de clasificar a este grupo de actores dentro de las lógicas de su actividad, es el de plantear su tipo de organización y oferta como: turismo comunitario, sin embargo esta expresión generalmente se encuentra asociada a espacios rurales y entornos de naturaleza, donde los proyectos de turismo buscan promover la conservación y el desarrollo sostenible (Longart, Wickens, Ocaña, \& Llugsha, 2017); este tipo de, propuestas análogamente similares, donde la comunidad es parte activa de la conservación y el desarrollo de su entorno, donde la protección de su entorno se vincula a un patrimonio de tipo cultural progresivamente van ganando terreno, dando paso a grupos que promueven un turismo comunitario urbano. Entre los grupos que destacan por el tiempo que desarrollan sus actividades y contar con una oferta consistente se encuentra: Caminos de San Roque, Colectivo Chakiñan y el Comité de Emprendedores de San Marcos (ver tabla 2).

Esto surge como una respuesta de los pobladores locales que ven tomados los atractivos turísticos del barrio, pero la comunidad no se beneficia de ningún tipo de ganancia; de esta forma el turismo es visto como una oportunidad de progreso para los pobladores de territorios donde se 
cuentan con recursos culturales y naturales, por otra parte, permite dinamizar la economía local concientizar respecto a procesos de conservación patrimonial. (Sicheri, Nolazco, \& Malvas, 2019). Sus propuestas han encontrado eco en distintas organizaciones que se han interesado en apoyar el desarrollo de un turismo en el que los miembros de la comunidad sean parte:

Gescultura, Museo Casa de El Alabado, Casa Gangotena, Mutualista Pichincha, Metropolitang Touring, Hotel Boutique San Marcos, Gobierno Provincial de Pichincha, Municipio de Quito.

Los colectivos y las asociaciones no solamente comparten un territorio similar, sino que enfrentan análogos conflictos, generados por la aplicación de los elementos establecidos en la normativa se dan a varios niveles, sin embargo las "limitaciones en la infraestructura, tal es el caso de las condiciones de arquitectura patrimonial”, son un factor que encarece el proceso de instalación de determinados giros de negocio (Quintana \& Salazar, 2018, p. 124).

Otra constante identificada radica en el hecho de que, por sus particularidades, al agruparse de forma comunitaria en un entorno urbano, no logran encontrar una figura dentro de la estructura legal que les permita trabajar dentro del turismo, de tal forma que han visto en el esquema de Economía Popular y Solidaria la posibilidad de legalizar sus actividades.

Las diferencias al interior de las organizaciones han provocado que varias personas abandonen los colectivos, quienes han desarrollado de forma paralela e individual actividades turísticas, convirtiéndose en competencia directa de sus vecinos.

Miembros de la asociación cuentan con locales mediante la figura de arriendo, por lo que en algunos casos los miembros abandonan el grupo debido a que deben dejar el local, esto se debe también al hecho de que los colectivos también son conformados por personas que no residen en el territorio, pero que desarrollan sus actividades económicas en el barrio.

\section{Conclusiones}

Los procesos de patrimonialización crean espacios escenificados (boutique) alrededor de los bienes patrimoniales, que son consumidos por los distintos actores del mercado turístico. Esto crea un efecto no deseado, la generación de espacios turistificados que dejan de ser pensados para los residentes.

La peatonización y las terrazas turísticas permiten tangibilizar la posición político institucional de la ciudad respecto a su Centro Histórico: patrimonio y turismo. El patrimonio cultural del centro si bien es presentado en el discurso como un elemento vivo, se muestra como un elemento de contemplación, sobre el cual los actores patrimoniales que habitan la centralidad pueden hacer uso del mismo en una relación en términos de consumo en la lógica de un mercado lúdico turístico cultural.

Los colectivos y asociaciones que buscan vincularse al turismo bajo una presencia de actores patrimoniales externos al barrio, contribuyen a consolidar la imagen del centro como un espacio turístico patrimonial de contemplación, donde las actividades económicas que se desarrollan. 
Si bien documento no profundiza en aspectos vinculados a la gentrificación en el territorio, se considera que existe pérdida de población residencial en el Centro Histórico, sin embargo, esta se encuentra vinculada a procesos de elitización que de forma progresiva han cambiado el tipo de actividades económicas en los barrios, pasando de una oferta diversa que satisfaga las necesidades de una población local, a una oferta de tipo turística que responde a la visión de una ciudad turística.

\section{Bibliografía}

Agencia Metropolitana de Control de Transporte Terrestre, Tránsito y Seguridad Vial. (2019, junio 4). Plan de movilidad (Desde la calle Sucre hasta la Bolívar). Recuperado de https://twitter.com/AMTQuito/status/1135887625884250112/photo/1

Carrión, F. (2003). Ciudad y centros históricos: Centros históricos y actores patrimoniales. En La Ciudad Inclusiva (pp. 129-152). Recuperado de https://repositorio.cepal.org//handle/11362/27821

Carrión, F. (2009). La centralidad histórica: Entre el nacionalismo del pasado (monumento) y el sentido social de hoy (centro vivo). Centro-h, (3), 7-12.

Delgadillo-Polanco, V. (2010). Aburguesamiento de barrios centrales, un proceso en expansión y mutación. Economía, sociedad y territorio, 10(34), 835-846.

Durán, L. (2014). La Ronda: Olvidar el barrio, recordar la calle. Recuperado de http://repositorio.flacsoandes.edu.ec/handle/10469/6120

Inostroza, G. V. (2008). Aportes para un modelo de gestión sostenible del turismo comunitario. Gestión turística, (10), 5-.

Instituto Metropolitano de Patrimonio. (2018, abril 20). Memorando $N^{\circ} I M P-D E P P-2018-3$.

Larrea, T. (2019). Turismo como alternativa urbana, análisis de la oferta turística emergente en el centro histórico de Quito durante el periodo 2012 -2017. Recuperado de http://repositorio.ute.edu.ec/xmlui/handle/123456789/18818

Llugsha, V. (2019). Turismo fronterizo. Aproximaciones a las asimetrías complementarias de un péndulo sobre las ciudades de frontera. En IV CONGRETUR, Impacto del Turismo en el desarrollo del Ecuador Libro de actas (pp. 14-23). Quito: Editorial Universitaria.

Longart, P., Wickens, E., Ocaña, W., \& Llugsha, V. (2017). A stakeholder analysis of a service learning project for tourism development in An Ecuadorian Rural Community. Journal of Hospitality, Leisure, Sport \& Tourism Education, 20, 87-100. https://doi.org/10.1016/j.jhlste.2017.04.002

Martínez del Vas, G. (2017). Proceso de configuración de un destino turístico y efectos de la política turística en la Región de Murcia. Cuadernos de Turismo, (40), 439-464.

Menchero, M. (2017). Las fortificaciones de Cartagena de Indias y su función turística en el turismo cultural de la ciudad. methaodos.revista de ciencias sociales, 5(1), 100-118.

Ministerio de Turismo del Ecuador. (2018). Catastro consolidado nacional de la planta turística del Ecuador.

Moreno, D. (2014). The road to equality in tourism Case study in Cartagena de India, Colombia. Memorias: Revista Digital de Historia y Arqueología desde el Caribe, (23), 26-51.

Oliveira, M. C. B. de, \& Pimentel, T. D. (2016). El Campo Turístico de la ciudad de Quito (Ecuador): Un análisis del capital de sus agentes y de la (posible) acción colectiva sobre las políticas de turismo como objeto de disputa. https://doi.org/10.14198/INTURI2016.12.09 
Prats, L. (2005). Concepto y gestión del patrimonio local. Cuadernos de Antropología Social, (21), 17-35.

Quintana, M. D., \& Salazar, D. A. (2018). Diagnóstico de establecimientos tradicionales del centro histórico de Quito-Ecuador, conforme a la normativa de zona especial turística. INNOVA Research Journal, 110-126. https://doi.org/10.33890/innova.v3.n8.2018.622

Quito Turismo. (2017). Boletín de Ocupación Hotelera n 129. Cifras a febrero, 2017.

Sicheri, L. G., Nolazco, F. A., \& Malvas, S. F. (2019). Turismo rural en el distrito de Chacas, departamento de Ancash, Perú. INNOVA Research Journal, 4(2), 13-20. https://doi.org/10.33890/innova.v4.n2.2019.1003

Verena, C. (2017). Los procesos de patrimonialización y turistificación en la legitimización de paisajes desiguales. Sociedade \& Natureza, 29, 195-208. 